The early stage of obstruction may present difficulties in diagnosis, but it is to be expected that a case which has progressed to extensive gangrene of the gut would have a history and clinical picture sufficiently clear to make the need for immediate surgical treatment obvious.

The case to be described had a history and symptoms which were so confusing and indefinite that a diagnosis of " acute abdomen" could not be made until the patient had been closely observed for forty-eight hours.

\section{History OF CASE}

The patient, a married woman, aged 45, was admitted into this hospital at 9 p.m. on November 18th, 1931, diagnosed by her doctor as a case of gastro-enteritis. She gave the following history

At about 11 o'clock the same morning she was sitting on a chair when she was suddenly seized with acute abdominal pain. The pain was general, and not localized to any particular point. She felt sick, and about five minutes later vomited some undigested food and yellow fluid. The pain was somewhat relieved after vomiting, and an hour later had entirely disappeared. She then had an attack of diarrhoea, the motions being green-coloured and very offensive, but, as far as she could tell, they did not contain any mucus or blood, nor did she experience any pain. The diarrinoea continued throughout the day, and she gradually became weaker and more exhausted. She had taken no food except a pieco of breal-and-butter and a cup of tea for breakfast, with cold water at intervals, until admission. The previous night she had had, with the rest of the household, some "fish and chips" for supper, but she was the only one to experience any ill effects afterwards. In her previous history she admitted having had a vaginal discharge twenty years ago. She had received several courses of injections, and had a blood test made a few years ago, which she had been told was positive. The history of previous venereal infection was, however, indefinite, and she gave no reliable information as to the treatment she had received. She had been operated upon for a prolapsed uterus nine or ten years ago, and after this she had been very constipated, with occasional attacks of diarrhoea. During the week before admission she had been constipated. There was no history of previous abdominal pain.

On admission her temperature was $97^{\circ} \mathrm{F}$., pulse 128 and very weak, and respirations 40. She was in a feeble and exhausted condition, with marked pallor, but had no abdominal pain. The tongue was dry and coated, teeth carious, breath very offensive, and she appeared toxic.

The abdomen moved with respiration; there was no visible peristalsis, and, except for slight tenderness in both iliac fossae on firm pressure, there was nothing further of note. The whole abdomen was soft, and no rigidity was discoverable. On examination per vaginam there was tenderness in both lateral fornices, and on the left side a firm, doughy mass could be made out. There was an offensive and purulent uterine discharge. Per rectum it was found that there was an incomplete fibrous stricture of the rectum, about half an inch above the junction of the anus and rectum. It completely encircled the rectum, but the little finger could pass through it. She was passing green and offensive motions, which were very relaxed, but contained no mucus or blood. Examination of heart and lungs revealed nothing abnormal. The pupils were concentric and equal, reacting normally to accommodation, but sluggishly to light. Refiexes were generally diminished, and the left knee-jerk was not obtained. Sensation was normal.

It was thought that her condition was a pvosalpinx, and that the diarrhoea was merely one of her usual attacks caused by the presence of the stricture. She was given hot vaginal douches of potassium permanganate $(1$ in 1,000$)$, a starch enema, and $1 / 4$ grain of morphine hypodermically. She passed a restless night, sleeping for short periods, but the diarrhoea continued. A half-hourly record of the pulse was kept, and by 7 o'clock the next morning the rate had decreased to 110 , and was much stronger. During the day (November 19th) the dirrinoea stopped, and until 11 p.m. on November 20 th her conlition improved, her pulse was better, and her temperature just below normal. After the initial dose no more morphine was given. At 11 p.m. on November 20th she collapsecl, with a pulse rate of 128 , temperature $97^{\circ} \mathrm{F}$., and began vomiting faecal material. It was decided to open the abdomen at once.

\section{Operation}

A left paramedian incision was made, and on opening the peritoneum it was found to be gangrenous. The peritoneal cavity contained some offensive serous and blood-stained fluid. The whole of the rectum, sigmoid, and the lower half of the descending colon were completely gangrenous, the upper limit boing fairly sharply defined. The remainder of the intestines were acutely inflamed. There was no volvulus, adhesion, growth, or other mechanical cause to account for the condition. It was found that the inferior mesenteric artery was pulseless, and that the blood supply to the affected gut was totally cut off, the artery with its branches being almost black in colour. A Paul's tube was tied into the transverse colon, a rubber drainage tube placed deep in the pelvis, and the wound clused. The patient, after draining well for five days, collapsed and died on November 26th.

\section{COMMENTARY}

The misleading feature of the case was the absence of a history and a clinical picture of sufficient severity to justify the findings at operation. Intestinal obstruction was considered as a diagnosis in view of the acute onset and the findings on rectal examination, but as she continually passed flatus, and the diarrhoea showed no signs of stopping, this was considered unlikely. The possibility of a tabetic crisis, the graver causes of gastro-enteritis, and lobar pneumonia (in view of the respiratory rate) had to be considered, but there was not sufficient evidence on which to make the diagnosis. The mass felt per vaginam was the gangrenous and distended gut, but there was no evidence of distension on percussion of sufficient magnitude to indicate the nature of the underlying condition. The diarrhoea, in conjunction with the incomplete stricture of the rectum, which was apparently specific in origin, accounted for the previous behaviour of the bowels. It was found impracticable to perform a postmortem examination, but the conclusions arrived ât were that an incorrect diagnosis had been made on the strength of clinical findings atypical of the condition found at operation.

We are indebted to Dr. R. Davidson, medical superintendent, St. Luke's Hospital, for permission to publish this case.

\section{IMMEDIATE PNEUMOCOCCAL TYPING} $\mathrm{BY}$

RICHARD R. ARMSTRONG, M.D., F.R.C.P. CATTLIN RESEARCH FELLOW, ST. BARTHOLOMFW'S MSPPTIL

The onset of winter and the reappearance of cases of severe primary pneumoccal pneumonia has afforded an opportunity for continuing the treatment by concentrated antipneumococcal serum described in the British Medical Journal a few months since. ${ }^{1}$ Determination of the type of pneumococcal infection is an essential preliminary to the administration of the specific therapeutic serum.

A rapid method by which pneumecoccal type could be decided within four hours was described in these columns a year ago." The method consisted in intraperitoneal inoculation of a mouse with the pneumonia patient's sputum. Four hours later the peritoneal contents were sampled by aspiration, the fluid obtained was mixed on a slide with type-specific agglutinating serums, and type was determined by microscopical examination of the fresh mixtures.

A number of tests made during the present season have proved, so far without exception, that type can be decided with ease and certainty by direct test on the patient's sputum, without recourse to mouse inoculation.

\section{Procedure}

The procedure is as follows:

A suitable fleck of sputum is selected. Three small samples of this are placed, equidistant, on a microscope 
slide, and numbered 1, 2, and 3 . Each sample is emulsified with four times its volume of the corresponding diagnostic serum, the addition of serum and emulsification being conveniently performed with a platinum loop. Coverglasses are applied, and the slide is set aside for a few minutes whilst a further sample from the selected fleck of sputum is smeared on a slide, fixed by heat, and stained by Gram's method. The general bacterial flora of the sputum and the number of pneumococci present are apparent at a glance in the stained film, which exactly represents the characters of the sputum samples selected for the diagnostic test. It is of special value to be informed in advance of these characters, for if the pneumococci are plentiful a positive result in the typing will be apparent at once; no time need be wasted in useless search, therefore, when, as in the case of a Group IV infection, there is no reaction.

The slide carrying the fresh emulsions of sputum and specific serums is now examined, using a 4 ocular, 1/6 objective, and plane mirror, the condenser being removed. Whereas the unstained pneumococci, when present in small numbers, are but just visible in the case of a negative test, the result in the case of a positive reaction is a conspicuous increase in the size of the individual pneumococcus, due to conjugation of coccus and homologous antibody. The enlarged cocci have a characteristic ground-glass appearance, with a highly refractive peripheral zone. A positive reaction is at times appreciable to the naked eye on holding the preparation to the light. The positive, as compared with its companions on the same slide (which serve as controls) is seen to be opalescent, due to the great increase in size of the " sensitized " cocci. When the pneumococci are thickly coated with sero-mucinous pneumonic secretion, the characteristic appearances develop more slowly as the specific serum soaks its way through. In such cases twenty minutes may elapse before full completion, although type may be distinguished much sooner by the change in those pairs which are floating free.

It is to be noted that type is decided independently of actual agglutination of the pneumococci, which is prevented, presumably, by the viscous nature of the sputum. These sputum elements are digested in the peritoneal cavity of the mouse, the cocci are freed, and multiply rapidly, so that agglutination takes place in presence of specific serum.

\section{COMMENTARY}

The results of the " direct test" have been confirmed in every case by mouse inoculation, and by the other more deliberate methods in common laboratory use. The direct method has proved trustworthy: its value lies in extreme simplicity and swiftness. The objection now disappears that pneumococcal type cannot be decided in general practice, since animal inoculation is no longer essential, and a special knowledge of bacteriological technique is not necessary.

Generally speaking, the later a case of pneumonia is examined, the more profuse the sputum and the more numerous the pneumococci; immediate typing as described is then a matter of minutes. Thus no time is lost in the intravenous administration of serum to the patient. On the other hand, early in pneumonia the sputum may be scanty and the pneumococci few. More care is required in making the test, and a second examination may be necessary. But at this stage a little delay does not materially affect the patient's chances of recovery.

Type may be decided with equal ease and speed by the direct method in the case of cerebro-spinal fluid, pus from empyemata, aural discharges, etc.

The specific monovalent diagnostic serums used were prepared in the laboratory by inoculation of young rabbits with typical strains of pneumococci. Similar reliable diagnostic serums are to be had from Messrs. Parke, Davis and Co.

The diagnosis and serum treatment of lobar pneumonia are being carried on in conjunction with my colleague R. Sleigh Johnson, who confirms the usefulness of the immediate diagnostic test. The Medical Research Council is assisting the inquiry.

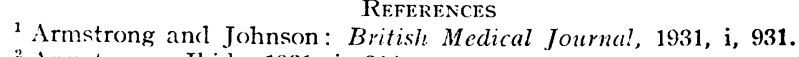
2Armstrong: Ibid., 1931, i, 214.

\section{A DIRECT METHOD OF TYPING PNEUMOCOCCI}

BY

\author{
W. R. LOGAN, M.D., F.R.C.P.ED., D.P.H. \\ BACTERIOLOGIST, ROYAL INFIRMARY, EDINBURGH \\ ANi) \\ J. T. SMEALI, M.C., M.B., CH.B., D.P.H. \\ SENIOR ASSISTANT BACTERIOLOGIST
}

In February, 1931, R. R. Armstrong ${ }^{1}$ drew attention to a phenomenon which was observed when the rapid typing of pneumococci by the mouse-peritoneal-exudate-agglutination method was carried out. Examining under the micrescope an unstained drop of peritoneal exudate mixed with undiluted serum, Armstrong observed that, in addition to agglutination with the specific serum, there was an increase in size of the pneumococci, and they became " very apparent as lanceolate bodies, surrounded by a clear, highly refractive capsular zone." This phenomenon was observed and fully described by Neufeld ${ }^{2}$ thirty years ago.

We have typed about 170 specimens of sputum from cases of lobar pneumonia during the past twelve months, using a variety of methods in each case, and always confirming the result of the rapid method by isolating the pneumococcus and determining its type when obtained in pure culture. We fully agree with Armstrong that his microscopical method is superior to other rapid methods, and particularly to the macroscopic-agglutination technique. It is only in the case of pure cultures free from cells that the macroscopic method has advantages, though even then the greater amount of serum required is of some economic importance.

The object of the present paper, however, is to show that the phenomenon referred to above makes it possible in some cases to recognize the type of pneumococcus by a direct test with the sputum emulsion itself. Many workers must have attempted a direct agglutination test with sputum emulsions. The consistency of sputum and the presence in many specimens of large numbers of other organisms make this impracticable in most cases, though not, as we have found, in all. Using the reaction just described, it is possible in some cases to diagnose the type confidently within a few minutes of receiving the specimen, although, of course, in other cases no definite opinion can be given.

\section{METHOD}

The technique is practically that described by Armstrong, the sputum emulsion being substituted for the mouse peritoneal exudate. A saline emulsion of the sputum is made. Four thin glass slides are marked I, II, III, and control, and a large loopful of the undiluted type serum is placed on the appropriate slide, a drop of salino being put on the control slide. The sputum emulsion is then taken up in a capillary pipette with a teat. A drop of the required size is placed beside each drop of serum 